\title{
NÃO NEUTRALIDADE FISCAL, GASTOS PÚBLICOS E AGENTES HETEROGÊNEOS
}

\author{
Victor Rodrigues de Oliveira ${ }^{a}$ \\ Fernando Motta Correia ${ }^{b}$

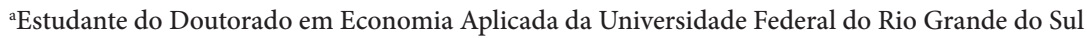 \\ (UFRGS).

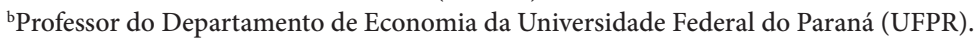

Artigo recebido em 21/08/2015 e aceito em 09/01/2016.

RESUMO: O objetivo deste estudo é verificar se a política fiscal é não neutra em um ambiente de endogeneidade dos gastos públicos com agentes heterogêneos. Considerou-se que a política fiscal pode ser conduzida por dois instrumentos, a saber, os impostos lump-sum e os gastos públicos. Para alcançar o objetivo proposto construiu-se um modelo novo keynesiano a partir dos trabalhos de Blanchard (1985) e Leith e Thadden (2008). Os resultados do sistema dinâmico indicam que as políticas monetária e fiscal devam ser ativas, o que denota para a não neutralidade da política fiscal.

PALAVRAS-CHAVE: política fiscal; gastos públicos; agentes não ricardianos.

CLASSIFICAÇÃO JEL: H30; H50; H31.

Correspondência para: Victor Rodrigues de Oliveira

Contato: victor5491@gmail.com 


\title{
NO FISCAL NEUTRALITY, PUBLIC SPENDING AND AGENTS HETEROGENEOUS
}

\begin{abstract}
The objective of this study is to verify if fiscal policy is not neutral in an endogeneity of public spending environment with heterogeneous agents. Fiscal policy is herein considered as being conducted by two instruments, namely the lump-sum taxes and public spending. To achieve the proposed objective it has been built up a new Keynesian model based on the works by Blanchard (1985) and Leith \& Thadden (2008). The results of the dynamic system indicate that monetary and fiscal policies should be active, which reveals the non-neutrality of fiscal policy.
\end{abstract}

KEY-WORDS: fiscal policy; government expenditures; non-Ricardian agents. 


\section{INTRODUÇÃO}

Um aspecto importante nas discussões sobre a coordenação entre as políticas fiscal e monetária é a definição do tipo de regime adotado pela autoridade monetária e a forma como o setor público financia seus deficit.

O uso do regime de metas de inflação como arcabouço operacional para o controle de preços torna as demais variáveis macroeconômicas subordinadas ao uso da taxa de juros nominal o principal instrumento na busca por uma meta de inflação programada pelo Banco Central. É o caso da oferta de moeda, que, nos modelos macroeconômicos tradicionais, é considerada exógena em favor da endogeneidade da taxa de juros. Agora, sob a hipótese de um regime do tipo metas de inflação, a política monetária, ao tornar a taxa de juros exógena no curto prazo, condiciona todas as demais variáveis a manter uma taxa de juros capaz de convergir a inflação para a meta desejada.

Por essa razão, o tipo de política fiscal numa economia que utiliza o regime de metas de inflação tem importantes consequências do ponto de vista dos resultados programados para a política monetária.

A literatura acerca dos efeitos econômicos dos deficit públicos busca explicar o impacto de diferentes regimes de política fiscal sobre o nível de preços bem como sobre a demanda agregada. O efeito econômico dos deficit públicos sobre o nível de preços acaba gerando efeitos sobre a demanda agregada dependendo das especificidades do regime de política fiscal, qual seja, regime ricardiano ou regime não ricardiano.

Em um ambiente de indeterminação, quando não se considera o papel ativo dos instrumentos fiscais, a dinâmica da dívida assume um papel fundamental para garantir o restabelecimento do equilíbrio de longo prazo. Como discutido por Favero e Giavazzi (2007), duas justificativas determinam a importância da dívida pública e da sua evolução na regra de decisão da autoridade fiscal: i) um feedback do nível da razão dívida pública/gasto público ou dívida pública/PIB é necessário para a estabilidade da dívida, a menos que a taxa de crescimento da economia seja exatamente igual ao custo médio de financiamento da dívida; e ii) a taxa de juros, uma variável central na transmissão de choques fiscais, depende da política monetária esperada e do prêmio de risco. Isto é, a taxa de juros pode ser afetada pela dinâmica da dívida, por exemplo, se um estoque crescente da mesma levanta a possibilidade de monetização no futuro ou, em caso extremo, de default da dívida. Assim, o impacto de um dado choque fiscal sobre a taxa de juros dependerá da trajetória (convergente, oscilante ou explosiva) da dívida pública. Disso decorre que a política fiscal deve ter um papel ativo.

Alguns trabalhos se preocupam em observar o papel da dívida pública, dos deficit do setor público e de regras fiscais na dinâmica de longo prazo em um ambiente de agentes 
ricardianos e não ricardianos. Em Blanchard (1985), toda a tributação é do tipo lump-sum, e a Equivalência Ricardiana é modelada por meio de um único parâmetro: a probabilidade de morte dos consumidores. Assim, quando essa probabilidade é positiva, há a presença de agentes não ricardianos e o horizonte temporal de decisão destes é menor do que o do governo. Leith e Thadden (2008) levam em conta a dependência do equilíbrio da dívida pública, de modo que tal variável deve ser levada em consideração na visualização de uma dinâmica de steady-state. Porém, em Blanchard (1985) e Leith e Thadden (2008) há uma limitante no que diz respeito às regras fiscais, tendo em vista que esses autores levam em conta apenas que as mudanças no nível de steady-state resultam de variações dos impostos, assumindo a hipótese de que os gastos do governo são exógenos e as mudanças no nível de steady-state resultam de variações dos impostos.

Mesmo em um ambiente de coordenação de política macroeconômica, onde a política fiscal por meio do gerenciamento do orçamento público sinalize uma política de subordinação à estabilidade de preços, há um ambiente de discricionariedade capturado a partir da exogeneidade da variável gasto público. Tal discricionariedade pode incorrer em problemas de inconsistência temporal da política econômica. Em alguns casos, embora a autoridade fiscal deixe clara a preocupação de manter um orçamento equilibrado, os indivíduos tendem a desconfiar dos anúncios das medidas fiscais, o que compromete as expectativas quanto aos deficit futuros.

Leith e Thadden (2008) desenvolvem uma estrutura analítica monetária e fiscal consistente ao identificar a dívida pública como variável determinante das condições de equilíbrio que, porém, faz uso de um grau de discricionariedade fiscal, uma vez que mantém exógena a variável gastos públicos.

O objetivo deste estudo é verificar se a política fiscal é não neutra em um ambiente de endogeneidade dos gastos públicos com agentes heterogêneos.

Além dessa introdução, o estudo será organizado da seguinte forma: na seção 2 será apresentada uma discussão entre política fiscal, agentes ricardianos e não ricardianos; a seção 3 apresenta o modelo empregado neste estudo; em seguida, o trabalho discutirá a existência do steady-state e as equações que determinam a dinâmica local em torno do steady-state; e na seção 5 são apresentados os resultados e as condições sob as quais o equilíbrio é estável. Finalmente, são apresentadas as principais conclusões.

\section{POLÍTICA FISCAL, AGENTES RICARDIANOS E NÃO RICARDIANOS: UM PANO DE FUNDO PARA O DEBATE}

A literatura acerca dos efeitos econômicos dos deficit públicos busca explicar o impacto de diferentes regimes de política fiscal sobre o nível de preços bem como sobre 
a atividade econômica. O efeito econômico dos deficit públicos sobre o nível de preços acaba gerando efeitos sobre a demanda agregada dependendo das especificidades do regime de política fiscal, qual seja, regime ricardiano ou regime não ricardiano.

De acordo com a Teoria Fiscal do Nível de Preços (TFNP), a política fiscal pode ser usada para selecionar a trajetória da inflação à medida que o governo tenha a habilidade de escolha do deficit público, assim, torne possível um equilíbrio compatível com o cenário fiscal programado (KOCHERLAKOTA e PHELAN, 1999). De acordo com McCallum (1998), a TFNP sustenta a ideia de que uma classe de regras políticas torna o nível de preços dependente da política fiscal e independente de variáveis monetárias.

Se o comportamento da política fiscal não leva em consideração as características das condições monetárias vigentes, o arcabouço operacional da política econômica pode se defrontar com a chamada dominância fiscal. Sargent e Wallace (1985) mostram a necessidade de a autoridade monetária evitar que a autoridade fiscal busque seu equilíbrio fiscal tendo como prática o financiamento do deficit público por meio da senhoriagem, na medida em que as autoridades monetárias podem estar sujeitas à perda do controle sobre o nível de preços ao gerar as receitas de Senhoriagem necessárias à manutenção da solvência fiscal.

Sob essa perspectiva, a forma como o deficit público é gerenciado torna-se um importante aspecto a ser analisado nas discussões sobre as interações entre as políticas monetária e fiscal. Para Leeper (1991), a política fiscal é ativa ou passiva, dependendo da receptividade do governo a choques da dívida pública. Desse modo, o gerenciamento do deficit público tem importantes efeitos sobre algumas variáveis macroeconômicas, entre as quais o nível de preços.

Se a estabilidade de preços é uma importante meta para a política econômica, o alcance dessa meta exige duas perguntas-chave segundo Christiano e Fitzgerald (2000): como a estabilidade de preços pode ser alcançada? O quanto a estabilidade de preços é desejada?

Como a TFNP propõe que os superavit primários do setor público evoluem independentemente da dívida do governo, temos, portanto, que o nível de preços de equilíbrio se elevará para assegurar a solvência fiscal (CANZONERI, CUMBY e DIBA, 1998). Essa é a base para o questionamento da visão tradicional acerca dos efeitos econômicos dos deficit públicos à luz do trabalho de Sargent e Wallace (1985). Para Rocha e Paschoalotto (2004), a abordagem tradicional encara o valor presente esperado dos superavit futuros como uma restrição ao comportamento do governo, que deve adotar uma política de gerenciamento de tributos e gastos que atenda à restrição orçamentária intertemporal do governo para qualquer nível de preços. Essa situação é conhecida como regime ricardiano. Na TFNP, o valor presente esperado dos superavit futuros é visto não como uma restrição e sim como uma condição de equilíbrio. Se o 
valor presente esperado dos superavit futuros é constante intertemporalmente, tem-se que o ajustamento deve ocorrer por meio do nível de preços e não pelo gerenciamento de tributos e gastos. Assim, nesse regime não ricardiano, a restrição orçamentária intertemporal do governo não é satisfeita para todos os níveis de preços, sendo o nível de preços de equilíbrio aquele que iguala o valor real das obrigações (nominais) do governo ao valor presente dos superavit futuros.

Se há uma inconsistência entre o tamanho do superavit primário esperado e o equilíbrio para o nível de preços e assumindo que não haja mudanças no nível de preços de equilíbrio, as famílias irão vislumbrar um aumento de sua riqueza, dado o aumento da dívida pública, proporcionando em consequência um aumento no consumo (WOODFORD, 1995). Com um aumento da demanda por bens, o nível de preços eleva-se, o que implica uma queda da riqueza real das famílias, forçando-as a reavaliar suas decisões de consumo na tentativa de equilibrar a demanda e a oferta de bens. Diante dessa análise, sob um regime não ricardiano, o ajuste de preços independe das condições de natureza monetária.

Portanto, os efeitos econômicos dos deficit públicos estão associados à resposta para uma das perguntas desenvolvidas por Christiano e Fitzgerald de como a estabilidade de preços pode ser alcançada, na medida em que, dependendo da característica do regime de política fiscal, ricardiano ou não ricardiano, o canal de transmissão entre as políticas monetária e fiscal pode ser distinto. Um importante aspecto associado a essa literatura é o grau de discricionariedade incorporado ao uso da política fiscal, uma vez que a variável gasto público é usualmente exógena na estrutura analítica dos modelos.

\section{MODELO}

No modelo que será apresentado, adotou-se uma especificação em que há a presença de dois instrumentos fiscais, isto é, além dos impostos, como convencionalmente utilizados por Blanchard (1985) e Leith e Thadden (2008), os gastos do governo são considerados endógenos. Com base nesses pressupostos a dinâmica da dívida tem impacto sobre a dinâmica do consumo agregado através da equação de Euler e, portanto, a primeira não é separável das demais condições de equilíbrio do modelo.

Como desenvolvido por Leith e Thadden (2008), o nível de endividamento permite capturar a não neutralidade da política fiscal por meio do efeito riqueza associado à equação de Euler. Além disso, como o processo de formação de capital é endógeno no modelo de Blanchard (1985), então existe uma relação entre o nível de steady-state da dívida e o efeito crowding out do capital. Este último efeito, por sua vez, afeta a taxa de juros real de steady-state, que é uma variável fundamental para a determinação do 
custo marginal das firmas. Como o custo marginal é um dos componentes que determinam a taxa de inflação corrente, a autoridade monetária deve considerar que as decisões de caráter fiscal afetarão a sua tomada de decisão. Esse mecanismo simples permite demonstrar que esses agentes econômicos estão interligados pela condução da dívida pública.

Considera-se que a política monetária segue uma regra de taxa de juros que especifica como esse instrumento reage aos desvios da inflação corrente em relação a uma meta de inflação e do produto corrente em relação ao produto potencial. Como a autoridade monetária está preocupada com os desvios dessas variáveis em relação a suas metas, tem-se um regime não conservador. Por outro lado, a autoridade fiscal tem a sua disposição dois instrumentos: os impostos lump-sum e o gasto do governo. A autoridade fiscal avalia como os desvios da dívida em relação a uma meta de dívida pública afetam os instrumentos a sua disposição. Essa especificação é uma modificação do trabalho de Leith e Thadden (2008).

\subsection{O PROBLEMA DO CONSUMIDOR}

Os consumidores são especificados como em Blanchard (1985), em que estão sujeitos a uma probabilidade constante de morte denotada por $\xi \geq 0$. Se essa probabilidade é positiva, então o horizonte de decisão efetiva dos agentes privados é menor do que o do governo. Quando $\xi=0$, tem-se uma economia com horizonte infinito. Neste estudo será analisado o caso em que há a presença de agentes heterogêneos, isto é, agentes ricardianos e agentes não ricardianos (hipótese de agentes heterogêneos).

Um consumidor nasce no período $j$. Em um período qualquer ele escolhe o consumo, $c_{s}^{j}$ e os saldos monetários reais, $m_{s}^{j}$, para maximizar sua função de utilidade intertemporal, assumindo como dados a preferência intertemporal, $\theta$, e a probabilidade constante de morte, $\xi$. Quando $\xi$ tende ao valor zero, todos os agentes são do tipo ricardiano, enquanto que todos os agentes são não ricardianos quando $\xi$ tende a um. Assume-se também que a oferta de trabalho é inelasticamente fixada em $n_{s}^{j}=1$. A utilidade esperada do consumidor representativo em $t$ pode ser expressa como segue:

$$
E_{t} U^{j}=\int_{t}^{\infty}\left[\ln c_{s}^{j}+\chi \ln m_{s}^{j}\right] e^{-(\xi+\theta)(s-t)} d s
$$

Em (1), $\chi$ representa a participação dos saldos monetários reais no portfólio do consumidor (cashless-limit quando $X \rightarrow 0$, como proposto por Woodford, 1998). Ad- 
mita que $U j$ é separável ${ }^{1}$ em $c$ e $m$ e que $u_{c}>0, u_{c c}<0, u_{m}>0<0$. O consumidor possui uma riqueza $a_{s}^{j}=k_{s}^{j}+b_{s}^{j}+m_{s}^{j}$, consistindo de capital físico $\left(k_{s}^{j}\right)$, de títulos do governo $\left(b_{s}^{j}\right)$ e de saldos monetários reais . A restrição orçamentária do consumidor representativo é dada por:

$$
d a_{s}^{j}=r_{s}\left(a_{s}^{j}-m_{s}^{j}\right)+\sigma a_{s}^{j}+w_{s}-\tau_{s}^{j}-c_{s}^{j}-\pi_{s} m_{s}^{j}+\Omega_{s}^{j}
$$

Em (2), $k_{s}^{j}$ e $b_{s}^{j}$ são substitutos perfeitos com taxa de retorno $r_{s} ; m_{s}^{j}$ deprecia-se à taxa de inflação $\pi_{s} ; \sigma a_{s}^{j}$ é o prêmio de risco pago pelas seguradoras, uma vez que os consumidores não viverão para sempre; $w$ s é o salário real; $\tau_{s}^{j}$ são os impostos lump-sum; e $\Omega_{s}^{j}$ é a participação nos lucros da produção de bens finais. A partir das equações (1) e (2), a condição de primeira ordem para o consumo é dada por $c_{s}^{j}=\frac{1}{\lambda_{s}^{j}}$ em que $\lambda_{s}^{j}$ denota a variável de co-estado da função hamiltoniana usada para solucionar o problema de otimização do consumidor. Os saldos reais satisfazem a seguinte condição:

$$
\frac{\chi}{m_{s}^{j}}=\lambda_{s}^{j}\left(r_{s}+\pi_{s}\right) \therefore m_{s}^{j}=\frac{\chi}{r_{s}+\pi_{s}} c_{s}^{j}
$$

Em (3) denota-se a equação da demanda por moeda. A variável de co-estado evolui ao longo do tempo de acordo com $d \lambda_{s}^{j}=-\left(r_{s}-\theta\right) \lambda_{s}^{j}$ e, portanto, a lei de movimento do consumo é $d c_{s}^{j}=\left(r_{s}-\theta\right) c_{s}^{j}$. Integrando a restrição orçamentária e impondo a condição de transversalidade com relação à riqueza não humana, isto é,

$$
\lim _{s \rightarrow \infty} a_{s}^{j} \cdot e^{-\int_{t}^{s}\left(r_{\mu}+\xi\right) d \mu}=0
$$

a restrição orçamentária intertemporal pode ser escrita como:

$$
\int_{t}^{\infty} c_{s}^{j} e^{-\int^{s}\left(r_{\mu}+\xi\right) d \mu} d s=\frac{1}{1+\chi}\left(a_{t}^{j}+h_{t}^{j}\right)
$$

Em (4) $h_{t}^{j}$ é a riqueza humana do indivíduo e é dada por:

\footnotetext{
1 Para uma observação pormenorizada sobre teoremas da separabilidade e suas implicações para teoria econômica, ver Ok (2007).
} 


$$
h_{t}^{j}=\int_{t}^{\infty}\left(w_{s}+\Omega_{s}^{j}-\tau_{s}^{j}\right) e^{-\int_{t}^{s}\left(r_{\mu}+\xi\right) d \mu} d s
$$

Integrando a lei de movimento do consumo, $c_{s}^{j}$, com relação a $t$ (para expressar $c_{s}^{j}$, como uma função de $c_{t}^{j}$,) obtém-se, a partir da restrição orçamentária intertemporal, a função de consumo individual dada por:

$$
c_{t}^{j}=\frac{\xi+\theta}{1+\chi}\left(a_{t}^{j}+h_{t}^{j}\right)
$$

\subsection{O COMPORTAMENTO AGREGADO DOS CONSUMIDORES}

O tamanho da população será normalizado e, portanto, a força de trabalho também será ao assumir-se que em qualquer momento no tempo uma nova coorte nasce e tem tamanho dado por $\xi$ (BLANCHARD, 1985). O tamanho total da população será sempre unitário dado que $\int_{-\infty}^{t} \xi e^{-\xi(t-j)} d j=1$, o que implica que $n t=1$ para todo $t$. Assim, a partir das equações (6) e (3) tem-se que:

$$
\left\{\begin{array}{l}
c_{t}=\frac{\xi+\theta}{1+\chi}\left(a_{t}+h_{t}\right) \\
m_{t}=\frac{\chi}{r_{t}+\pi_{t}} c_{t}
\end{array}\right.
$$

Em (7), $a_{t}=k_{t}+b_{t}+m_{t}$. As leis de movimento de $h_{t}$ e de $a_{t}$ podem ser escritas como:

$$
\left\{\begin{array}{l}
d h_{t}=\left(r_{t}+\xi\right) h_{t}-w_{t}+\tau_{t}-\Omega_{t} \\
d a_{t}=r_{t} a_{t}+w_{t}-(1+\chi) c_{t}+\Omega_{t}-\tau_{t}
\end{array}\right.
$$

Diferenciando $c_{t}$ com relação ao tempo e substituindo $h_{t}$ é possível estabelecer a lei de movimento do consumo como segue:

$$
d c_{t}=\left(r_{t}-\theta\right) c_{t}-\xi \frac{\xi+\theta}{1+\chi} a_{t}
$$


A partir da equação (9) observa-se que se $\xi>0$, a taxa de crescimento do consumo individual $\left(r_{t}-\theta\right)$ excede a taxa de crescimento do consumo agregado. Isto é, esse resultado é decorrente do efeito geracional, uma vez que os agentes com alto nível de riqueza não humana são substituídos por agentes novos sem nenhuma riqueza. Dessa forma, é possível introduzir um efeito riqueza puro no consumo diferentemente do obtido por meio da equivalência ricardiana, e esse efeito pode ser capturado em único parâmetro, $\xi$.

\subsection{FIRMAS}

O modelo considera a presença de dois tipos de firmas, que serão apresentados a seguir.

\subsubsection{FIRMAS DE ALUGUEL DE CAPITAL}

Há um continuum de firmas que acumulam capital para alugar para os produtores de bens finais. Assuma que it denote o investimento real dessas firmas, obtido por um mix de bens finais que é idêntico ao do consumo privado. Assuma também que o capital se deprecia a uma taxa ${ }^{\mathrm{TM}}>0$. $\mathrm{O}$ estoque de capital evolui de acordo com a seguinte lei de movimento:

$$
d k_{t}=i_{t}-\delta k_{t}
$$

As firmas de aluguel de capital são de propriedade das famílias. O retorno do capital é igual a uma taxa livre de risco, $r_{t}$, se a taxa de aluguel do capital $p_{t}^{k}$ cobrado do setor de bens finais satisfizer a condição de lucro zero: $p_{t}^{k}=r_{t}+\delta$.

\subsubsection{PRODUTORAS DE BENS FINAIS}

Assume-se que os bens finais são produzidos por firmas em um mercado de concorrência imperfeita, que estão sujeitas às restrições impostas por contratos do tipo Calvo, isto é, as firmas são capazes de mudar os preços com probabilidade $\alpha$. A probabilidade de mudança de preços é definida no intervalo $(0,1)$, isto é, uma fração dos preços dos bens permanece fixa em cada período de tempo (Calvo, 1983). Para este estudo considerou-se o modelo com preços pré-determinados e ajustamento alternado, de forma que em cada período $50 \%$ das firmas irão fixar seus preços para os próximos dois 
$\operatorname{anos}^{2}$. As firmas são indexadas por $z$ e produzem de acordo com uma função de produção do tipo Cobb-Douglas:

$$
y(z)_{t}=n(z)_{t}^{\gamma} k(z)_{t}^{1-\gamma}
$$

Em (11), os insumos são adquiridos em um mercado competitivo. Escrevendo a função hamiltoniana tem-se o problema das firmas produtoras de bens finais:

$$
H=p_{t}^{k} k(z)+w_{t} n(z)+\lambda\left(y(z)_{t}-n(z)_{t}^{\gamma} k(z)_{t}^{1-\gamma}\right)
$$

A minimização de custos implica que a combinação de capital e de trabalho empregada pela firma é dada por:

$$
\frac{k(z)_{t}}{n(z)_{t}}=\frac{k_{t}}{n_{t}}=k_{t}=\frac{1-\gamma}{\gamma} \frac{w_{t}}{p_{t}^{k}}
$$

A equação (13) é igual para todas as firmas porque elas são tomadoras de preço no mercado de insumos.

Assumindo-se retornos constantes de escala, tem-se que o custo marginal é dado por:

$$
M C=\left(p_{t}^{k}\right)^{1-\gamma} w_{t}^{\gamma} \gamma^{-\gamma}(1-\gamma)^{\gamma-1}
$$

No período $t$ a firma representativa tem a seguinte demanda:

$$
y(z)_{t}=\left(\frac{p(z)_{t}}{p_{t}}\right)^{-\rho} y_{t}
$$

Em (15), $y t$ é a demanda total por bens finais, $\rho>1$ denota a elasticidade constante de demanda ${ }^{3}$.

2 Nesse modelo com ajustamento alternado são feitas três hipóteses: i) os preços são fixados em dois períodos, mas podem ser diferentes em cada um dos períodos; ii) em cada período, metade dos produtores irá fixar os seus preços para os próximos dois anos; e iii) em cada período, metade das firmas terá que adotar os preços fixados no contrato estabelecido há dois períodos atrás e o restante adota os preços fixados no período anterior.

3 O índice de preços pode ser aproximado como segue:

$p_{t}=\left[\int_{0}^{1} p(z)_{t}^{1-\rho}\right]^{\frac{1}{1-\rho}}$ 
A função objetivo da firma é dada por:

$$
V_{t}=\int_{t}^{\infty}\left\{\left(\frac{p(z)_{t}}{p_{t}}\right)^{1-\rho} y_{s}-M C_{s}\left(\frac{p(z)_{t}}{p_{t}}\right)^{-\rho} y_{s}\right\} e^{-\int^{s}\left(r_{\mu}+\xi\right) d \mu} d s
$$

O preço ótimo decorrente do problema de otimização é dado por:

$$
p(z)_{t}=\frac{\int_{t}^{\infty}\left\{\rho\left(\frac{1}{p_{s}}\right)^{-\rho} M C_{s} y_{s}\right\} e^{-\int_{t}^{s}\left(r_{\mu}+\alpha\right) d \mu} d s}{\int_{t}^{\infty}\left\{(\rho-1)\left(\frac{1}{p_{s}}\right)^{1-\rho} y_{s}\right\} e^{-\int_{t}^{s}\left(r_{\mu}+\alpha\right) d \mu} d s}
$$

Vale observar que a equação de determinação de preços, (17), representa uma generalização da regra de marcação de preços do tipo mark-up: $p(z)_{t} / p=\rho /(\rho-1) M C$. O índice de preços agregado que prevalece no período $t$ é uma média ponderada de preços, onde os pesos refletem a proporção desses preços que ainda estão em vigência, isto é,

$$
p_{t}=\left(\int_{-\infty}^{t}(\alpha(p(z) s))^{1-\rho} e^{-\alpha(t-s)} d s\right)^{\frac{1}{1-\rho}}
$$

O lucro agregado do setor de bens finais pode ser escrito como:

$$
\Omega_{t}=\int_{-\infty}^{t} \alpha\left\{\left(\frac{p(z)_{s}}{p_{t}}\right)^{1-\rho}-M C_{t}\left(\frac{p(z)_{s}}{p_{t}}\right)^{-\rho}\right\} y_{t} e^{-\alpha(t-s)} d s
$$

\subsection{GOVERNO}

Assuma que $\ell_{t}$ denote as responsabilidades agregadas (reais) do setor público consistindo de saldos monetários reais e de títulos do governo, $\ell_{t}=m_{t}+b_{t}$. A dinâmica de $\ell_{t}$ pode ser expressa como:

$$
d \ell_{t}=r_{t} \ell_{t}-\left(r_{t}+\pi_{t}\right) m_{t}+g_{t}-\tau_{t}=r_{t} \ell_{t}-\chi c_{t}+g_{t}-\tau_{t}
$$


Em (20), $g_{t}$ denota o gasto do governo. Diferentemente de Leith e Thadden (2008), que consideram $g_{t}=g>0$, isto é, exógeno, este estudo considera que o gasto do governo é endógeno.

Assumem-se as seguintes regras para a política monetária e a política fiscal:

1. Com relação à política monetária considera-se uma meta de inflação $\pi^{\star}$. Esse argumento da função de reação da autoridade monetária é diferente daquele observado em Leith e Thadden (2008). Dada a possibilidade de alteração dos preços, a autoridade monetária tem, no curto prazo, influência sobre a taxa real de juros, $r_{t}$, por meio de operações de open market. Como se adotou a hipótese de Woodford (1998), podem se ignorar as rendas oriundas de senhoriagem. Assim, a Regra de Taylor modificada apresenta a seguinte estrutura:

$$
r_{t}=r+\beta_{1}\left(\pi_{t}-\pi^{*}\right)+\beta_{2}\left(y_{t}-y^{*}\right)
$$

Em (21), $r$ é o nível de steady-state da taxa real de juros, $y^{*}$ é o produto potencial e $-1 \leq \beta \leq 1$. Observe-se que no caso de cashless-limit, tem-se que $\ell_{t} \rightarrow b_{t}$.

2. A política fiscal segue uma regra que objetiva estabilizar $b_{t}$ em uma meta $b^{\star}$. Observe que as decisões de política monetária afetam a restrição orçamentária da autoridade fiscal por meio de dois canais: i) a taxa real de juros influencia diretamente o retorno do capital privado, que é alugado para as firmas de bens finais pelas famílias; e ii) a taxa real de juros também influencia o valor real da dívida pública, que pode ser aproximada por $b=\frac{\tau-g}{r}$, ou seja, o valor real dos títulos públicos em circulação deve ser igual ao valor presente dos superavit fiscais primários, como em Díaz-Giménez et al. (2008). Nesse caso têm-se duas regras de condução da política fiscal:

$$
\left\{\begin{array}{l}
\tau_{t}=\tau+\beta_{3}\left(b_{t}-b^{*}\right) \\
g_{t}=g+\beta_{4}\left(y_{t}-y^{*}\right)+\beta_{5}\left(b_{t}-b^{*}\right)
\end{array}\right.
$$

Em (22), $\tau_{t}$ de nota os impostos lump-sum no período $t$; $\tau$ é o valor de steady-state dos impostos; e $g$ é o nível de steady-state do gasto público, $0 \leq \beta_{3} \leq 1,-1 \leq . \beta_{4} \leq 0$ e $-1 \leq \beta_{5} \leq 0$. 


\section{STEADY-STATE}

\subsection{CONDIÇÕES DE EQUILÍBRIO}

As condições que caracterizam um equilíbrio dinâmico no nível agregado podem ser resumidas de acordo com o modelo apresentado anteriormente, como segue:

$$
\begin{aligned}
& d c_{t}=\left(r_{t}-\theta\right) c_{t}-\xi \frac{\xi+\theta}{1+\chi}\left(k_{t}+b_{t}\right) \\
& d b_{t}=r_{t} b_{t}+g_{t}-\tau_{t} \\
& r_{t}=r+\beta_{1}\left(\pi_{t}-\pi^{*}\right)+\beta_{2}\left(y_{t}-y^{*}\right) \\
& \tau_{t}=\tau+\beta_{3}\left(b_{t}-b^{*}\right) \\
& g_{t}=g+\beta_{4}\left(y_{t}-y^{*}\right)+\beta_{5}\left(b_{t}-b^{*}\right) \\
& d k_{t}=i_{t}-\delta k_{t} \\
& y_{t}=k_{t}^{1-\gamma} \\
& k_{t}=\frac{1-\gamma}{\gamma} \frac{w_{t}}{r_{t}+\delta} \\
& M C=\left(r_{t}+\delta\right)^{1-\gamma} w_{t}^{\gamma} \gamma^{-\gamma}(1-\gamma)^{\gamma-1} \\
& p(z)_{t}=\frac{\int_{t}^{\infty}\left\{\rho\left(\frac{1}{p_{s}}\right)^{-\rho} M C_{s} y_{s}\right\} e^{-\int_{t}^{s}\left(r_{\mu}+\alpha\right) d \mu} d s}{\int_{t}^{\infty}\left\{(\rho-1)\left(\frac{1}{p_{s}}\right)^{1-\rho} y_{s}\right\} e^{-\int_{t}^{s}\left(r_{\mu}+\alpha\right) d \mu} d s} \\
& p_{t}=\left(\int_{-\infty}^{t}\left(\alpha\left(p(z)_{s}\right)\right)^{1-\rho} e^{-\alpha(t-s)} d s\right)^{\frac{1}{1-\rho}}
\end{aligned}
$$

As condições de equilíbrio requerem que duas equações sejam satisfeitas: (i) condição de market clearing, $y_{t}=c_{t}+g_{t}+i_{t}$; (ii) identidade da renda, $y_{t}=w_{t}+\left(r_{t}+\delta\right) k_{t}+\Omega_{t}$. 


\subsection{EXISTÊNCIA DO STEADY-STATE}

Considerando $\chi \rightarrow$ observa-se que $\ell_{t} \rightarrow b_{t}$. Além disso, no steady-state $p(z)=p$ e normalize $p=1$. A partir de (32), o mark-up implica que $\frac{\rho-1}{\rho}=M C$. Combinando (30) e (31) e considerando-se que, os preços dos insumos podem ser definidos como:

$$
\begin{gathered}
w(k)=w=\frac{\rho-1}{\rho} \gamma k^{1-\gamma} \\
r(k)=r=\frac{\rho-1}{\rho} \frac{(1-\gamma)}{k^{\gamma}}-\delta
\end{gathered}
$$

Onde $w(k)$ e $r(k)$ representam as expressões da produtividade marginal quando a economia torna-se perfeitamente competitiva $(\rho \rightarrow \infty)$.

Combinando a versão de steady-state da equação de consumo (23) com a restrição (24) tem-se que:

$$
c=\xi(\xi+\theta) \frac{k+\frac{\tau-g}{r}}{r-\theta}
$$

Por outro lado, a limitação de recursos no steady-state é dada por:

$$
c=k^{1-\gamma}-g-\delta k
$$

Se $\xi>0$, então no steady-state $r>0$, dado que os consumidores não ricardianos são mais "impacientes" do que os consumidores ricardianos, uma vez que os primeiros não viverão para sempre. A partir das equações (36) e (37) e um pouco de álgebra é possível estabelecer a seguinte condição de equilíbrio:

$$
0<g<\gamma y(\mathrm{~A})
$$

Conforme a condição de equilíbrio (A), considere-se uma situação inicial de equilíbrio orçamentário, isto é, $g=\tau \Leftrightarrow b=0$. Então, a economia é caracterizada por dois steady-states com níveis positivos de consumo, de capital e de produto, quando o nível do gasto público não é muito alto - ver Figura 1(a). 
Figura 1 - Configuração do steady-state
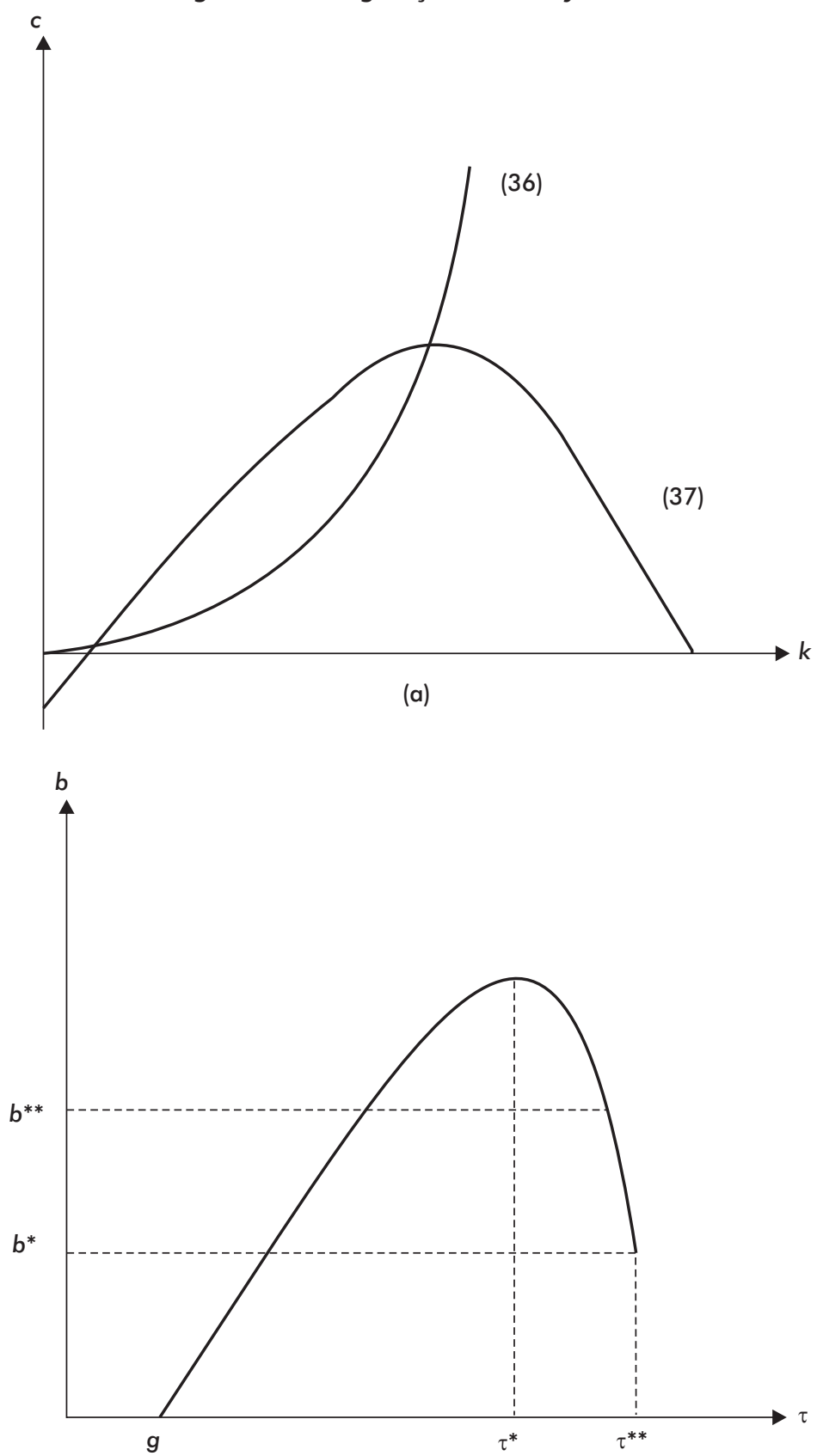

(b)

Fonte: Elaboração própria. 
Um aumento de $\tau$ gera um nível positivo de dívida, que em ambos os steady-states afeta o consumo e o capital através do efeito riqueza. Em outras palavras, os títulos do governo são percebidos como riqueza líquida pelos consumidores atualmente vivos, já que a carga tributária, apoiando esses títulos, é parcialmente suportada pelos membros das gerações futuras. Observe que quando $\tau$ aumenta, o superavit primário também aumenta. Além disso, a taxa real de juros aumenta, uma vez que o efeito riqueza conduz a uma diminuição do capital físico (crowding out). Esses dois movimentos tornam ambíguo o efeito líquido sobre o steady-state da dívida, que é dado pelo valor descontado dos superavit primários (ou seja, $b=(\tau-g) / r$ ). Assim, considere-se a condição (A), isto é, $0<g=\tau<\gamma y$. Ao se variar $\tau$ e manter $g$ constante existe um intervalo de steady-states eficientes caracterizados por $\tau \in\left(g, \tau^{*}\right) \Rightarrow b \in\left(0, b^{\star *}\right)$. De forma semelhante, existe um intervalo de steady-states ineficientes caracterizados por $\tau \in\left(\tau^{*}, \tau^{* *}\right) \Rightarrow b \in\left(b^{*}, b^{* *}\right)$. Esses resultados podem ser visualizados na Figura $1(\mathrm{~b})$.

\section{DINÂMICA}

Combinando as equações (23)-(33) e a condição de market clearing, a dinâmica local em torno do steady-state é caracterizada por um sistema dinâmico em $c_{t}, b_{t}, k_{t}$ e $\pi_{t}$. Dada a não linearidade do modelo considerou-se uma aproximação de primeira ordem. Assume-se que $\hat{x}_{t}=\frac{x_{t}-x}{x}$ e $d \hat{x}_{t}=\frac{\partial \hat{x}_{t}}{\partial t}$ para $x=b, c, k$ e $\pi$. A partir das equações diferenciais (23), (24) e (28) e das funções de reação da autoridade fiscal e da autoridade monetária, equações (25)-(27), é fácil obter-se as aproximações de $\hat{c}_{t}, \hat{b}_{t}$ e $\hat{k}_{t}$ :

$$
\begin{gathered}
d \hat{c}_{t}=(r-\theta) \hat{c}_{t}-\xi(\xi+\theta) \frac{k}{c} \hat{k}_{t}-\xi(\xi+\theta) \frac{b}{c} \hat{b}_{t}+\beta_{1}\left(\pi_{t}-\pi^{*}\right)+\beta_{2}\left(y_{t}-y^{*}\right) \\
d \hat{b}_{t}=\left(r-\beta_{3}-\beta_{5}\right) \hat{b}_{t}+\beta_{1}\left(\pi_{t}-\pi^{*}\right)+\beta_{2}\left(y_{t}-y^{*}\right) \\
d \hat{k}_{t}=-\frac{c}{k} \hat{c}_{t}+\left[(1-\gamma) \frac{y}{k}-\delta\right] \hat{k}_{t}-\beta_{5} \frac{g}{k} \hat{b}_{t}
\end{gathered}
$$

Observe-se que nas equações (38) e (39) foi incluída a diferença da taxa de juros real com relação ao seu valor de steady-state. Esse termo permite analisar como alterações da política monetária afetam a dinâmica do consumo e da dívida pública.

Para se obter a aproximação $d \pi_{t}$ considere que $\widehat{p(z)_{t}}=\left(p(z)_{t}-p(z)\right) / p(z)$. Da equação (32) é possível obter $\widehat{p(z)})_{t}$ a partir da Regra de Leibnitz, como se segue: 


$$
\begin{gathered}
\widehat{p(z)_{t}}=\int_{t}^{\infty}(r+\alpha)\left[\hat{p}_{s}+\widehat{M C}_{s}\right] e^{-(r+\alpha)(s-t)} d s \\
d \widehat{p(z)_{t}}=(r+\alpha) \widehat{p(z)_{t}}-(r+\alpha)\left(\hat{p}_{t}+\widehat{M C}_{t}\right)=(r+\alpha)\left(\widehat{p(z)_{t}}-\hat{p}_{t}-\widehat{M C}_{t}\right) \\
\hat{p}_{t}=\int_{-\infty}^{t} \alpha p(z)_{s} e^{-\alpha(t-s)} d s
\end{gathered}
$$

De forma semelhante, a partir da equação (33) é possível obter $\hat{p}_{t}$, como se segue:

$$
\left.d \hat{p}_{t}=\alpha(\widehat{p(z)})_{t}-\hat{p}_{t}\right)
$$

Assim, a inflação será positiva sempre que o ajustamento de preços for superior ao nível de preços médio. Para obter a dinâmica da inflação é necessário encontrar $\widehat{M C}_{t}$ combinando-se as equações (25), (30) e (31) e, então:

$$
\widehat{M C}_{t}=\hat{p}_{t}^{k}+\gamma \hat{k}_{t}=\gamma \hat{k}_{t}+\beta_{1}\left(\pi_{t}-\pi^{*}\right)+\beta_{2}\left(y_{t}-y^{*}\right)+(r+\delta)
$$

Combinando-se as equações (44) e (45) obtém-se a dinâmica da inflação, como segue:

$$
\begin{gathered}
\left.d \pi_{t}=\alpha\left((r+\alpha)(\widehat{p(z)})-\hat{p}_{t}-\widehat{M C}_{t}\right)-\alpha\left(\widehat{p(z)_{t}}-\hat{p}_{t}\right)\right)=r \pi_{t}-\alpha(\alpha+r) \widehat{M C}_{t} \\
=r \pi_{t}-\alpha(\alpha+r)\left[\gamma \hat{k}_{t}+\beta_{1}\left(\pi_{t}-\pi^{*}\right)+\beta_{2}\left(y_{t}-y^{*}\right)+(r+\delta)\right]
\end{gathered}
$$

Para avaliar a estabilidade do sistema formado pelas equações (38), (39), (40) e (46) monta-se a matriz jacobiana, $j$, como se segue:

$$
\left[\begin{array}{l}
d \hat{c}_{t} \\
d \hat{k}_{t} \\
d \hat{b}_{t} \\
d \pi_{t}
\end{array}\right]=J\left[\begin{array}{l}
\hat{c}_{t} \\
\hat{k}_{t} \\
\hat{b}_{t} \\
\pi_{t}
\end{array}\right]
$$

Em (47), a matriz $j$ é assim definida:

$$
J=\left[\begin{array}{cccc}
r-\theta-\xi(\xi+\theta) k / c & -\xi(\xi+\theta) b / c & \beta_{1} \\
-c / k(1-\gamma) 1 / k^{\gamma}-\delta & -\beta_{5} g / k & 0 \\
0 & 0 & r-\beta_{3}-\beta_{5} & \beta_{1} \\
0 & -\alpha(\alpha+r) \gamma & 0 & r-\alpha(\alpha+r) \beta_{1}
\end{array}\right]
$$


Para o sistema descrito em (48) ser estável é necessário e suficiente que $\operatorname{Det}(J)>0$ e $\operatorname{Tr}(J)<0$.

$$
\operatorname{Det}(J)=\left[(r-\theta)\left(\eta^{0}+\eta^{1}\right)+\left(\frac{c}{k}\right) \eta^{2}\right]
$$

Em (49), tem-se que:

$$
\begin{gathered}
\eta^{0}=\left(r-\beta_{3}-\beta_{5}\right)\left(r-\alpha(\alpha+r) \beta_{1}\right)\left((1-\gamma) \frac{1}{k^{\gamma}}-\delta\right), \eta^{1}=\alpha(\alpha+r) \gamma \beta_{1} \beta_{5} \frac{g}{k}, \\
\eta^{2}=\xi(\xi+\theta)\left(r-\beta_{3}-\beta_{5}\right)\left(r-\alpha(\alpha+r) \beta_{1}\right) \frac{k}{c}+\alpha(\alpha+r) \gamma \beta_{1}\left(\xi(\xi+\theta) \frac{b}{c}+\left(r-\beta_{3}-\beta_{5}\right)\right) \\
\operatorname{Tr}(J)=\omega^{0}-\omega^{F}-\omega^{M} \beta_{1}
\end{gathered}
$$

Depreende-se que:

São condições para $\operatorname{Tr}(J)$ ser negativo:

1. A primeira hipótese é que a autoridade monetária assume uma posição ativa na determinação da taxa real de juros de curto prazo, isto é, $\beta_{1}>0$. Assim, desvios da inflação corrente em relação à meta de inflação têm impacto positivo sobre a taxa de juros. Essa condição demonstra que somente o princípio de Taylor não garante a estabilidade local do sistema apresentado em (48), ou seja, as condições para a estabilidade dependem, além dos parâmetros da política monetária, dos aspectos fiscais, dado o efeito riqueza associado à equação de Euler.

2. A segunda hipótese diz respeito à autoridade fiscal, de forma que é possível diferenciar três situações: i) a dinâmica da política fiscal pode ser conduzida pelo ajuste dos impostos lump-sum e, assim, como o parâmetro $\beta_{3}$ é positivo, o termo $\omega^{F}$ também será; ii) a autoridade fiscal pode utilizar o gasto público como o único instrumento fiscal. Nesse caso, para o equilíbrio ser estável é necessário que haja coordenação de política econômica, de forma que $\left|\beta_{5}\right|>\left|\beta_{1}\right|$; e iii) a política fiscal pode ser conduzida pelos gastos públicos e pelos impostos. Aqui, a condição necessária para a estabilidade é que $\left|\beta_{3}\right|>\left|\beta_{5}\right|$, ou seja, o efeito de desvios da dívida pública com relação à meta para a mesma, $b^{\star}$, é maior sobre os impostos. Nesse modelo com agentes heterogêneos a hipótese é que a proporção de agentes ricardianos é maior do que a proporção de agentes não ricardianos, assim, aumentos da dívida pública deverão ser financiados, em parte, por aumento dos impostos. A partir disso, observa-se que na presença de dívida pública a política fiscal deve assumir um papel ativo. 
3. A terceira hipótese está relacionada à proposição de Woodford (1998). Isto é, adotando-se o limite inferior da taxa real de juros e considerando-se a presença de agentes heterogêneos, é possível inferir-se que os agentes privados irão dar maior preferência para o consumo presente, de forma que o parâmetro $\theta$ tenderá ao valor um. Como os agentes não viverão indefinidamente, o limite do termo $(r-\theta)$ tende a zero. Uma vez que a taxa de juros tende ao seu valor mínimo, a produtividade marginal do capital também tenderá a esse valor, de forma a manter válida a equação (35), e pela lei dos rendimentos marginais decrescentes, a economia é caracterizada por um alto estoque de capital físico.

São condições para $\operatorname{Det}(J)$ ser positivo:

1. A primeira condição é que $r-\alpha(\alpha+r) \beta_{1} \geq 0$. Dadas as hipóteses com relação a e $\beta_{1}$, essa condição sempre é satisfeita.

2. A segunda condição supõe que a política fiscal é ativa, isto é, $r>\beta_{3}+\beta_{5}$, como proposto por Leith e Thadden (2008). Observe que o caráter ativo da política fiscal dependerá do instrumento utilizado. Essa hipótese permite destacar o fato de a inserção da dívida pública no modelo capturar a não neutralidade da política fiscal.

\section{CONSIDERAÇÕES FINAIS}

A política fiscal, de forma geral, assume um papel secundário quanto ao seu uso como instrumento de estabilização macroeconômica. A política monetária é vista como o instrumento ideal para determinar a trajetória ótima do nível de atividade econômica, por meio da manipulação da taxa de juros de curto prazo através de operações, principalmente, de open market. Esse tratamento assimétrico é adequado quando se considera que todos os agentes são ricardianos. Assim, é possível separar as decisões de política monetária e de política fiscal. Entretanto, quando o horizonte temporal dos agentes privados difere do horizonte do governo, essas decisões não podem ser separadas. Nesse caso, a política fiscal deve assumir um papel ativo.

A partir disso, o objetivo deste trabalho foi verificar se a política fiscal é não neutra em um ambiente de endogeneidade dos gastos públicos com agentes heterogêneos. Para tanto, foi proposto um modelo novo keynesiano a partir de Blanchard (1985) e Leith e Thadden (2008). Entretanto, diferentemente desses autores, considerou-se que a autoridade fiscal dispõe de dois instrumentos fiscais, isto é, a política fiscal pode ser conduzida por meio dos impostos lump-sum ou do gasto do público ou ambos. A inserção de agentes não ricardianos é modelada através de mudanças na probabilidade de morte. Quando essa probabilidade é positiva, a Equivalência Ricardiana deixa de 
ser válida, uma vez que o horizonte temporal dos agentes privados é diferente do horizonte do governo. Além disso, nesse caso a dinâmica da dívida pública assume um papel relevante na determinação do equilíbrio local.

Os resultados da estrutura analítica apresentada podem apontar para a viabilidade do uso de regras em um ambiente de coordenação de política macroeconômica. Os principais resultados encontrados indicaram que a existência de um equilíbrio estável está associada a uma política monetária e uma política fiscal ativas, desde que haja no componente de endogeneidade dos gastos públicos um comprometimento dos gastos públicos com os desvios da dívida pública em face de uma meta de dívida pública pré-estabelecida. As análises de estabilidade apontam para a imposição de uma estrutura orçamentária capaz de conter o ambiente de discricionariedade que pode ser caracterizado a partir do uso indefinido do gasto público. A maior parte da literatura considera a variável gasto público de natureza exógena, tornando-a altamente discricionária. A imposição de uma função reação para os gastos públicos, ao tornar tais gastos dependentes dos desvios da dívida, fez surgir um canal de transmissão entre as variáveis fiscais e a necessidade do uso de regras fiscais associadas com o comprometimento pela estabilidade do grau de endividamento. Uma vez que há a necessidade de se impor a condição $\left|\beta_{3}\right|>\left|\beta_{5}\right|$, ou seja, que os impostos devem reagir aos desvios da dívida em velocidade superior à reação dos gastos frente aos mesmos desvios, tal resultado gera um comprometimento por parte da autoridade fiscal em um ambiente de coordenação de política macroeconômica, uma vez que regimes monetários do tipo metas de inflação estão associados ao conhecido tripé de política macroeconômica "metas de inflação, câmbio flexível e estabilidade fiscal". Perceba que o resultado $\left|\beta_{3}\right|>\left|\beta_{5}\right|$ impõe um comprometimento por parte da autoridade fiscal frente a todo arcabouço de política macroeconômica ao gerar comprometimento de tal autoridade econômica com a não explosividade da dívida pública.

\section{REFERÊNCIAS}

BLANCHARD, O. Debt, deficits, and finite horizons. Journal of Political Economy, v. 93, n. 2, p. 223-247, 1985.

CALVO, G. Staggered prices in a utility-maximizing framework. Journal of Monetary Economics, v. 12, n. 3, p. 983-998, 1983.

CANZONERI, M. B., CUMBY, R. E.; DIBA, B. Is the price level determined by the needs of fiscal solvency? NBER Working Paper, n. 6471, mar. 1998.

CHRISTIANO, L. J.; FITZGERALD, T. J. Understanding the fiscal theory of the price level. NBER Working Paper, n. 7668, abr. 2000. 
FAVERO, C.; GIAVAZZI, F. Debt and the effects of fiscal policy. Working Paper, Federal Reserve Bank of Boston, n. 4, 2007.

KOCHERLAKOTA, N.; PHELAN, C. Explaining the fiscal theory of the price level. Federal Reserve Bank of Minneapolis Quarterly Review, Fall, 1999.

LEEPER, E. Equilibria under "active" and "passive" monetary and fiscal policies. Journal of Monetary Economics, v. 27, n. 1, p. 129-147, 1991.

LEITH, C.; THADDEN, L. V. Monetary and fiscal policy interactions in a New Keynesian model with capital accumulation and non-Ricardian consumers. Journal of Economic Theory, v. 140, n. 1, p. 279-313, 2008.

MCCALLUM. B. T. Indeterminacy, bubbles and the fiscal theory of price level determination. NBER Working Paper, n. 6456, 1998.

OK, E. A. Real analysis with economic applications. Princeton: Princeton University Press, 2007.

ROCHA, F. F.; PASCHOALOTTO, E. Teoria fiscal do nível de preços: um teste para a economia brasileira no período 1966/2000. Pesquisa e Planejamento Econômico, v. 34, n. 3, 2004.

SARGENT, T. J.; WALLACE, N. Some unpleasant monetarist arithmetic. Federal Reserve Bank of Minneapolis Quarterly Review, n. 9, p. 15-31, Winter, 1985.

WOODFORD, M. Doing without money: controlling inflation in a post-monetary world. Review of Economic Dynamics, v. 1, n. 1, p. 173-219, 1998.

WOODFORD, M. Price level determinacy without control of a monetary aggregate. Carnegie Rochester Conference Series on Public Policy, v. 43, p. 1-46, dez. 1995. 


\section{APÊNDICE A}

$\mathrm{O}$ agente representativo maximiza o valor presente do fluxo de utilidade do consumo, $c_{s}^{j}$, e do saldo monetário real, $m_{s}^{j}$, ao longo de sua vida finita, descontado pela taxa de preferência intertemporal $\theta$ e pela probabilidade constante de morte $\xi$. Como argumenta Blanchard (1985), não há, portanto, nenhum problema de inconsistência temporal com relação à condição inicial do problema. Assim, o problema do agente representativo consiste, portanto, em maximizar

$$
E_{t} U^{j}=\int_{t}^{\infty}\left(\ln c_{s}^{j}+\chi \ln m_{s}^{j}\right) e^{-(\xi+\theta)(s-t)} d s
$$

Este está sujeito às seguintes restrições:

$$
d a_{s}^{j}=r_{s}\left(k_{s}^{j}+b_{s}^{j}\right)+\sigma a_{s}^{j}+w_{s}-\tau_{s}^{j}-c_{s}^{j}-\pi_{s} m_{s}^{j}+\Xi_{s}^{j} a_{s}^{j}(0)=0
$$

O hamiltoniano, $H$, desse problema, é dado por:

$$
H=\left(\operatorname{In} c_{s}^{j}+\chi \ln m_{s}^{j}\right) e^{-(\xi+\theta)(s-t)}+\lambda_{s}^{j}\left[r_{s}\left(a_{s}^{j}-m_{s}^{j}\right)+\sigma a_{s}^{j}+w_{s}-\tau_{s}^{j}-c_{s}^{j}-\pi_{s} m_{s}^{j}+\Xi_{s}^{j}\right]
$$

Na equação acima, $\lambda_{s}^{j}$ é a variável de coestado. As condições de primeira ordem são as seguintes:

$$
\begin{gathered}
\frac{\partial H}{\partial c_{s}^{j}}=\frac{1}{c_{s}^{j}}-\lambda_{s}^{j}=0 \\
\frac{\partial H}{\partial m_{s}^{j}}=\frac{\chi}{m_{s}^{j}}-\lambda_{s}^{j}\left(r_{s}+\pi_{s}\right)=0 \\
d \lambda_{s}^{j}=(\xi+\theta) \lambda_{s}^{j}-\frac{\partial H}{\partial a_{s}^{j}}=(\xi+\theta) \lambda_{s}^{j}-\lambda_{s}^{j}\left[r_{s}-\sigma\right]=-\lambda_{s}^{j}\left(r_{s}-\theta\right) \\
\frac{\partial H}{\partial \lambda_{s}^{j}}=r_{s}\left(k_{s}^{j}+b_{s}^{j}\right)+\sigma a_{s}^{j}+w_{s}-\tau_{s}^{j}-c_{s}^{j}-\pi_{s} m_{s}^{j}+\Xi_{s}^{j}=d a_{s}^{j}
\end{gathered}
$$
Da equação (51) decorre que $c_{s}^{j}=\frac{1}{\lambda_{s}^{j}}$. Rearranjando a equação (52) é possível
mostrar que: 


$$
\begin{gathered}
\frac{\chi}{m_{s}^{j}}-\lambda_{s}^{j}\left(r_{s}+\pi_{s}\right)=0 \\
\frac{\chi}{m_{s}^{j}}=\lambda_{s}^{j}\left(r_{s}+\pi_{s}\right) \\
m_{s}^{j}=\frac{\chi}{\lambda_{s}^{j}\left(r_{s}+\pi_{s}\right)} \Leftrightarrow m_{s}^{j}=\frac{\chi}{\left(r_{s}+\pi_{s}\right)} c_{s}^{j}
\end{gathered}
$$

A solução ótima deve satisfazer a seguinte condição de transversalidade:

$$
\lim _{s \rightarrow \infty} a_{s}^{j} e^{-\int_{t}^{s}\left(r_{\mu}+\xi\right) d \mu}=0
$$

Essa condição afirma que a trajetória ótima deve ser tal que o valor presente da riqueza deve ser igual a zero quando o tempo decresce de forma ilimitada. Caso contrário, o agente poderia aumentar seu bem estar diminuindo a acumulação de riqueza, isto é, a acumulação de capital físico, de títulos públicos e de saldos monetários reais e consumindo seus recursos. 


\section{APÊNDICE B}

Derivando $c_{t}$ com relação ao tempo e considerando que $h_{t}=c_{t}-a_{\mathrm{t}}$, obtém-se:

$$
\begin{aligned}
& \frac{\partial}{\partial}=d c_{t}=\frac{\xi+\theta}{+\chi}\left(d a_{t}+d h_{t}\right) \\
& \frac{\partial}{\partial}=\frac{\xi+\theta}{+\chi}\left[r_{t} a_{t}+w_{t}-(+\chi) c_{t}+\Xi_{t}-\tau_{t}+\left(r_{t}+\xi\right) h_{t}-w_{t}+\tau_{t}-\Xi_{t}\right] \\
& \frac{\partial}{\partial}=\frac{\xi+\theta}{+\chi}\left[r_{t} c_{t}+\xi c_{t}-(+\chi) c_{t}-\xi a_{t}\right] \\
& \frac{\partial}{\partial}=\left(r_{t}-\theta\right) c_{t}-\xi \frac{\xi+\theta}{+\chi} a_{t}
\end{aligned}
$$




\section{APÊNDICE C}

A firma representativa minimiza o custo total de produção sujeito à função de produção (ou tecnologia). Assim, considerando $p_{t}^{k}$ como o preço do capital $\left(k_{z, t}\right)$ no período $t$ e $w_{t}$ como o salário real no período $t$ que remunera o fator trabalho $\left(n_{z, t}\right)$, a função hamiltoniana, $\mathcal{H}$, pode ser escrita como segue:

$$
\mathcal{H}=p_{t}^{k} k_{z, t}+w_{t} n_{z, t}+\lambda\left(y_{z, t}-n_{z, t}^{\gamma} k_{z, t}^{1-\gamma}\right)
$$

A minimização de custos implica que as condições de primeira ordem são as seguintes:

$$
\begin{gathered}
\frac{\partial H}{\partial k_{z, t}}=p_{t}^{k}-\lambda(1-\gamma) n_{z, t}^{\gamma} k_{z, t}^{-\gamma}=0 \\
\frac{\partial H}{\partial n_{z, t}}=w_{t}-\lambda \gamma n_{z, t}^{\gamma-1} k_{z, t}^{1-\gamma}=0 \\
\frac{\partial H}{\partial \lambda}=y_{z, t}-n_{z, t}^{\gamma} k_{z, t}^{1-\gamma}=0
\end{gathered}
$$

Resolvendo as equações (57) e (58) para $\lambda$ e igualando-se os resultados obtém-se que a combinação de $k_{z, t}$ e $n_{z, t}$ é dada por:

$$
\begin{aligned}
& \frac{p_{t}^{k}}{(1-\gamma) n_{z, t}^{\gamma} k_{z, t}^{-\gamma}}= \frac{w_{t}}{\gamma n_{z, t}^{\gamma-1} k_{z, t}^{1-\gamma}} \\
& \frac{k_{z, t}}{n_{z, t}}=\frac{k_{t}}{n_{t}}=k_{t}=\frac{1-\gamma}{\gamma} \frac{w_{t}}{p_{t}^{k}}
\end{aligned}
$$

A equação (60) é a mesma para todas as firmas, uma vez que elas são tomadoras de preço no mercado de insumos. Observe-se que se considerou a hipótese de oferta de trabalho exogenamente fixada em $n_{t}=1$.

Substituindo as expressões para $n_{t}$ e $k_{t}$ obtidas na equação (60) na restrição da firma representativa, os valores ótimos de $n_{t}$ e $k_{t}$ seguem abaixo:

$$
n_{t}=\frac{y}{\left(\frac{1-\gamma}{\gamma} \frac{w_{t}}{p_{t}^{k}}\right)^{1-\gamma}}
$$




$$
k_{t}=\frac{y}{\left(\frac{\gamma}{1-\gamma} \frac{p_{t}^{k}}{w_{t}}\right)^{\gamma}}
$$

Substituindo as equações (61) e (62) na função de custo total, CT, da firma representativa, obtém-se

$$
\begin{aligned}
& C T=p_{t}^{k} k_{t}+w_{t} n_{t} \\
& =p_{t}^{k} \frac{y}{\left(\frac{\gamma}{1-\gamma} \frac{p_{t}^{k}}{w_{t}}\right)^{\gamma}}+w_{t} \frac{y}{\left(\frac{1-\gamma}{\gamma} \frac{w_{t}}{p_{t}^{k}}\right)^{1-\gamma}} \\
& =y\left[p_{t}^{k}\left(\frac{1-\gamma}{\gamma} \frac{w_{t}}{p_{t}^{k}}\right)^{\gamma}+w_{t}\left(\frac{\gamma}{1-\gamma} \frac{p_{t}^{k}}{w_{t}}\right)^{1-\gamma}\right] \\
& =y\left(p_{t}^{k}\right)^{1-\gamma}\left(w_{t}\right)^{\gamma}\left(\frac{1}{(\gamma)^{\gamma}(1-\gamma)^{1-\gamma}}\right)
\end{aligned}
$$

A partir da equação (63), o custo marginal, MC, pode ser aproximado por:

$$
\frac{\partial C T}{\partial y}=M C=\left(p_{t}^{k}\right)^{1-\gamma}\left(w_{t}\right)^{\gamma}(\gamma)^{-\gamma}(1-\gamma)^{\gamma-1}
$$




\section{APÊNDICE D}

Considere novamente a equação (16):

$$
V_{t}=\int_{t}^{\infty}\left[\left(\frac{p_{z, t}}{p_{t}}\right)^{1-\rho} y_{s}-M C_{s}\left(\frac{p_{z, t}}{p_{t}}\right)^{-\rho} y_{S}\right] e^{-\int^{s}\left(r_{\mu}+\xi\right) d \mu} d s
$$

Derivando a equação acima com relação ao preço praticado pela firma, $p z$, $t$, pela Regra de Leibniz, é possível mostrar que:

$$
\begin{aligned}
& \frac{d V_{t}}{d p_{z, s}}=\frac{d}{d p_{z, s}} \int_{t}^{\infty}\left[\left(\frac{p_{z, t}}{p_{t}}\right)^{1-\rho} y_{s}-M C_{s}\left(\frac{p_{z, t}}{p_{t}}\right)^{-\rho} y_{s}\right] e^{-\int f_{t}^{s}\left(r_{\mu}+\xi\right) d \mu} d s \\
& =\int_{t}^{\infty} \frac{\partial}{\partial p_{z, s}}\left[\left(\frac{p_{z, t}}{p_{t}}\right)^{1-\rho} y_{s}-M C_{s}\left(\frac{p_{z, t}}{p_{t}}\right)^{-\rho} y_{s}\right] e^{-\int f_{t}^{s\left(r_{\mu}+\xi\right) d \mu}} d s \\
& =\int_{t}^{\infty}\left[-(1-\rho)\left(\frac{1}{p_{s}}\right)^{-\rho} y_{s}-M C_{s} y_{s} \rho\left(\frac{1}{p_{s}}\right)^{-\rho-1}\right] e^{-\iint_{t}^{s}\left(r_{\mu}+\xi\right) d \mu} d s \\
& =\int_{t}^{\infty}\left[-(1-\rho)\left(\frac{1}{p_{s}}\right)^{-\rho} y_{s}\right] e^{-\int\left(r_{\mu}+\xi\right) d \mu} d s \\
& -\int_{t}^{\infty}\left[M C_{s} y_{S} \rho\left(\frac{1}{p_{s}}\right)^{-\rho-1}\right] e^{-\int f_{t}^{s}\left(r_{\mu}+\xi\right) d \mu} d s \\
& =\int_{t}^{\infty}\left[(\rho-1)\left(\frac{1}{p_{s}}\right)^{-\rho} y_{s}\right] e^{-\int_{t}^{s}\left(r_{\mu}+\xi\right) d \mu} d s \\
& -\int_{t}^{\infty}\left[M C_{s} y_{S} \rho\left(\frac{1}{p_{s}}\right)^{-\rho-1}\right] e^{-\int\left(r_{\mu}+\xi\right) d \mu} d s \\
& =\frac{1}{p_{s}}\left\{\int_{t}^{\infty}\left[(\rho-1)\left(\frac{1}{p_{s}}\right)^{-\rho} y_{s}\right] e^{-\int_{t}^{s}\left(r_{\mu}+\xi\right) d \mu} d s\right\} \\
& -\frac{1}{p_{s}}\left\{\int_{t}^{\infty}\left[M C_{s} y_{s} \rho\left(\frac{1}{p_{s}}\right)^{-\rho-1}\right] e^{-\int\left(_{\mu}+\xi\right) d \mu} d s\right\} \\
& p_{z, t}=\frac{\int_{t}^{\infty}\left[\rho\left(\frac{1}{p_{s}}\right)^{-\rho} M C_{s} y_{s}\right] e^{-\int_{t}^{s}\left(r_{\mu}+\alpha\right) d \mu} d s}{\int_{t}^{\infty}\left[(\rho-1)\left(\frac{1}{p_{s}}\right)^{1-\rho} y_{s}\right] e^{-\int_{t}^{s}\left(r_{\mu}+\alpha\right) d \mu} d s}
\end{aligned}
$$




\section{APÊNDICE E}

Da equação (13) decorre que

$$
k_{t}=\frac{(1-\gamma)}{\gamma} \frac{w_{t}}{(r+\delta)} \Rightarrow r+\delta=\frac{(1-\gamma)}{\gamma} \frac{w}{k}
$$

e considerando que $M C=\frac{\rho-1}{\rho}$, tem-se que

$$
\begin{aligned}
\frac{\rho-1}{\rho} & =\left(\frac{(1-\gamma)}{\gamma} \frac{w}{k}\right)^{1-\gamma} w^{\gamma}(\gamma)^{-\gamma}(1-\gamma)^{\gamma-1} \\
& =(r+\delta)^{1-\gamma} w^{\gamma}(\gamma)^{-\gamma}(1-\gamma)^{\gamma-1} \\
& =w(\gamma)^{-1}(k)^{\gamma-1} \Rightarrow w(k)=w=\frac{\rho-1}{\rho} \gamma(k)^{1-\gamma}
\end{aligned}
$$

Reescrevendo a equação (13) como segue

$$
k_{t}=\frac{(1-\gamma)}{\gamma} \frac{w_{t}}{(r+\delta)} \Rightarrow w=\frac{\gamma}{(1-\gamma)}(r+\delta) k
$$

e considerando que $M C=\frac{\rho-1}{\rho}$, tem-se que

$$
\begin{aligned}
\frac{\rho-1}{\rho} & =(r+\delta)^{1-\gamma}\left(\frac{\gamma}{(1-\gamma)}(r+\delta) k\right)^{\gamma}(\gamma)^{-\gamma}(1-\gamma)^{\gamma-1} \\
& =(r+\delta)^{1-\gamma} k^{\gamma}(r+\delta)^{\gamma} \frac{\gamma^{\gamma}}{(1-\gamma)^{\gamma}} \gamma^{-\gamma}(1-\gamma)^{\gamma-1} \\
& =\frac{(r+\delta)}{(1-\gamma)} k^{\gamma} \Rightarrow r(k)=r=\frac{\rho-1}{\rho} \frac{(1-\gamma)}{k^{\gamma}}-\delta
\end{aligned}
$$




\section{APÊNDICE F}

Derivando a equação que estabelece o consumo no steady-state com relação a $k$, isto é, $\frac{d c}{d k}$, tem-se que: $h_{t}^{j}$

Derivando a equação que estabelece limitação de recursos no steady-state com relação à $k$, isto é, $\frac{d c}{d k}$, tem-se que:

$$
\begin{aligned}
& \frac{d c}{d k}=(1-\gamma) \frac{1}{k^{\gamma}}-\delta>0 \\
& \frac{d^{2} c}{d k^{2}}=-\gamma(1-\gamma) \frac{1}{k^{\gamma+1}}<0
\end{aligned}
$$




\section{APÊNDICE G}

A partir das equações (23), (24) e (29) e das funções de reação da autoridade fiscal e da autoridade monetária, obtêm-se as aproximações de $\hat{c}_{t}, \hat{b}_{t}$ e $\hat{k}_{t}$ como segue:

$$
\begin{gathered}
d c_{t}=\left(r_{t}-\theta\right) c_{t}-\xi \frac{\xi+\theta}{1+\chi}\left(k_{t}+b_{t}\right) \\
d \hat{c}_{t}=\left(r_{t}-\theta\right) \hat{c}_{t}-\xi(\xi+\theta)\left(\hat{k}_{t}+\hat{b}_{t}\right) \\
=(r-\theta) \hat{c}_{t}-\xi(\xi+\theta) \frac{k}{c} \hat{k}_{t}-\xi(\xi+\theta) \frac{b}{c} \hat{b}_{t}+\beta_{1}\left(\pi_{t}-\pi^{*}\right)+\beta_{2}\left(y_{t}-y^{*}\right) \\
d b_{t}=r_{t} b_{t}+g_{t}-\tau_{t} \\
=r_{t} b_{t}+g+\beta_{4}\left(y_{t}-y^{*}\right)+\beta_{5}\left(b_{t}-b^{*}\right)-\tau-\beta_{3}\left(b_{t}-b^{*}\right)+\beta_{1}\left(\pi_{t}-\pi^{*}\right)+\beta_{2}\left(y_{t}-y^{*}\right) \\
=\left(r_{t}+\beta_{5}-\beta_{3}\right) b_{t}+\left(\beta_{4}+\beta_{2}\right)\left(y_{t}-y^{*}\right)+\left(\beta_{3}-\beta_{5}\right) b^{*}+g-\tau+\beta_{1}\left(\pi_{t}-\pi^{*}\right) \\
d \hat{b}_{t}=\left(r-\beta_{3}+\beta_{5}\right) \hat{b}_{t}+\beta_{1} \pi_{t}+\left(\beta_{4}+\beta_{2}\right)(1-\gamma) \frac{y}{k} \hat{k}_{t}-\left(\beta_{4}+\beta_{2}\right) y^{*}+\left(\beta_{3}-\beta_{5}\right) b^{*}+g-\tau-\beta_{1} \pi^{*} \\
d k_{t}=k_{t}^{1-\gamma}-c_{t}-g_{t} \\
d \hat{k}_{t}=\left((1-\gamma) \frac{y}{k}-\delta\right) \hat{k}_{t}-\left(\frac{c}{k}\right) \hat{c}_{t}-\left(\beta_{5} \frac{g}{k}\right) \hat{b}_{t}
\end{gathered}
$$

Observe que nas equações (68) e (69) foi incluída a diferença da taxa de juros real com relação ao seu valor de steady-state. Esse termo permite analisar como alterações da condução da política monetária afetam a dinâmica do consumo e da dívida pública.

Para obter a aproximação de $d \pi_{t}$, considere que $\hat{p}_{z . t}=\left(p_{z, t}-p_{z}\right) / p_{z}$. É possível obter $\hat{p}_{t}$ a partir da Regra de Leibnitz, como segue:

$$
\begin{gathered}
\hat{p}_{z . t}=\int_{t}^{\infty}(r+\alpha)\left(\hat{p}_{s}+\widehat{M C}_{s}\right) e^{-(r+\alpha)(s-t)} d s \\
d \hat{p}_{z . t}=(r+\alpha) \hat{p}_{z . t}-(r+\alpha)\left(\hat{p}_{t}+\widehat{M C}_{t}\right)=(r+\alpha)\left(\hat{p}_{z . t}-\hat{p}_{t}-\widehat{M C}_{t}\right)
\end{gathered}
$$

De forma semelhante, a partir da equação (18) é possível obter $\hat{p}_{t}$, como segue:

$$
\begin{gathered}
\hat{p}_{t}=\int_{-\infty}^{t} \alpha p_{z, s} e^{-\alpha(t-s)} d s \\
d \hat{p}_{t}=\alpha\left(\hat{p}_{z . t}-\hat{p}_{t}\right)
\end{gathered}
$$


Assim, a inflação será positiva sempre que o ajustamento de preços for superior ao nível de preços médio. Para obter a dinâmica da inflação é necessário encontrar $\widehat{M C}_{t}$ como segue:

$$
\widehat{M C}_{t}=\hat{p}_{t}^{k}+\gamma \hat{k}_{t}=\gamma \hat{k}_{t}+\beta_{1}\left(\pi_{t}-\pi^{*}\right)+\beta_{2}\left(y_{t}-y^{*}\right)+(r+\delta)
$$

Combinando as equações (74) e (75) obtém-se a dinâmica da inflação, como segue:

$$
\begin{aligned}
d \pi_{t}= & \alpha\left[(r+\alpha)\left(\widehat{p(z)}-\hat{p}_{t}-\widehat{M C}_{t}\right)-\alpha\left(\widehat{p(z)_{t}}-\hat{p}_{t}\right)\right] \\
= & r \pi_{t}-\alpha(\alpha+r) \widehat{M C}_{t} \\
= & r \pi_{t}-\alpha(\alpha+r)\left[\gamma \hat{k}_{t}+\beta_{1}\left(\pi_{t}-\pi^{*}\right)+\beta_{2}\left(y_{t}-y^{*}\right)+(r+\delta)\right] \\
= & \left(r-\alpha(\alpha+r) \beta_{1}\right) \pi_{t}-\alpha(\alpha+r) \gamma \hat{k}_{t} \\
& -\alpha(\alpha+r)\left[-\beta_{1} \pi^{*}+\beta_{2}\left(y_{t}-y^{*}\right)+(r+\delta)\right]
\end{aligned}
$$




\section{APÊNDICE H}

A matriz jacobiana é expressa como segue:

$$
\left[\begin{array}{l}
d \hat{c}_{t} \\
d \hat{k}_{t} \\
d \hat{b}_{t} \\
d \pi_{t}
\end{array}\right]=\left[\begin{array}{l}
\phi_{c}(k, c, b, \pi) \phi_{k}(k, c, b, \pi) \phi_{b}(k, c, b, \pi) \phi_{\pi}(k, c, b, \pi) \\
\varphi_{c}(k, c, b, \pi) \varphi_{k}(k, c, b, \pi) \varphi_{b}(k, c, b, \pi) \varphi_{\pi}(k, c, b, \pi) \\
\Omega_{c}(k, c, b, \pi) \Omega_{k}(k, c, b, \pi) \Omega_{b}(k, c, b, \pi) \Omega_{\pi}(k, c, b, \pi) \\
\Theta_{c}(k, c, b, \pi) \Theta_{k}(k, c, b, \pi) \Theta_{b}(k, c, b, \pi) \Theta_{\pi}(k, c, b, \pi)
\end{array}\right]
$$

O traço da matriz (77) segue abaixo, considerando que $\delta \rightarrow 0$ :

$$
\begin{aligned}
|z z| & =r+(r-\theta)-\alpha(\alpha+r) \beta_{1}+\left(r-\beta_{3}+\beta_{5}\right)+(1-\gamma) / k^{\gamma} \\
& =2 r-\alpha(\alpha+r) \beta_{1}-\beta_{3}+\beta_{5}
\end{aligned}
$$

O determinante da matriz (77) é obtido pelo Teorema de Laplace, como segue, considerando que $\delta \rightarrow 0$ :

$$
\begin{aligned}
& |\operatorname{Det}(z z)|=\alpha(\alpha+r) \gamma\left|\begin{array}{ccc}
r-\theta & -\xi(\xi+\theta) b / c \beta_{1} \\
-c / k & -\beta_{5} g / k & 0 \\
0 & r-\beta_{3}+\beta_{5} & \beta_{1}
\end{array}\right| \\
& +\left(r-\alpha(\alpha+r) \beta_{1}\right)\left|\begin{array}{ccc}
r-\theta & -\xi(\xi+\theta) k / c & -\xi(\xi+\theta) b / c \\
-c / k & (1-\gamma) / k^{\gamma} & -\beta_{5} g / k \\
0 & \left(\beta_{4}+\beta_{2}\right)(1-\gamma) y / k & r-\beta_{3}+\beta_{5}
\end{array}\right| \\
& =\alpha(\alpha+r) \gamma\left[\beta_{1}\left|\begin{array}{cc}
-c / k & -\beta_{5} g / k \\
0 & r-\beta_{3}+\beta_{5}
\end{array}\right|+\beta_{1} \mid \begin{array}{cc}
r-\theta-\xi(\xi+\theta) b / c \\
-c / k & -\beta_{5} g / k
\end{array}\right] \\
& -\left(r-\alpha(\alpha+r) \beta_{1}\right)\left(\beta_{4}+\beta_{2}\right)(1-\gamma) y / k\left|\begin{array}{ll}
r-\theta-\xi(\xi+\theta) b / c \\
-c / k & -\beta_{5} g / k
\end{array}\right| \\
& +\left(r-\alpha(\alpha+r) \beta_{1}\right)\left(r-\beta_{3}+\beta_{5}\right)\left|\begin{array}{ll}
r-\theta-\xi(\xi+\theta) k / c \\
-c / k & (1-\gamma) / k^{\gamma}
\end{array}\right| \\
& =\frac{1}{k}\left\{-\alpha(\alpha+r) \gamma \beta_{1}\left(r-\beta_{3}+\beta_{5}\right) c\right. \\
& \left.+\left[(r-\theta) \beta_{5} g+\xi(\xi+\theta) b\right]\left[\left(r-\alpha(\alpha+r) \beta_{1}\right)\left(\beta_{4}+\beta_{2}\right)(1-\gamma) y-\alpha(\alpha+r) \beta_{1}\right]\right\}
\end{aligned}
$$

